\title{
Prevalencia de miopía y factores de riesgo asociados en estudiantes de medicina en Monterrey
}

\section{Prevalence of myopia and associated risk factors in medical students in Monterrey}

\author{
Christian G. Cavazos-Salias ${ }^{1 \#, ~ N a t a l i a ~ M o n t e m a y o r-S a l d a n ̃ a ~}{ }^{1 \#, ~ L u c y ~ S a l u m-R o d r i ́ g u e z " 1, ~}$ \\ Juan E. Villarreal-Del Moral ${ }^{1}$ y Manuel Garza-Leon ${ }^{1,2 *}$ \\ ${ }^{1}$ Departamento de Ciencias Clínicas, División de Ciencias de la Salud, Universidad de Monterrey, San Pedro Garza García, Nuevo León; ${ }^{2}$ Hospital \\ Dr. Luís Sánchez Bulnes, Asociación para Evitar la Ceguera en México, Ciudad de México. México \\ "Ambos autores contribuyeron de forma similar, por lo que tienen el mismo reconocimiento.
}

\section{Resumen}

Antecedentes y objetivo: La miopía se ha convertido en un problema de salud pública, por lo que es importante conocer su prevalencia en diferentes grupos poblacionales, los estudiantes de medicina son considerados un grupo de riesgo. Nuestro objetivo fue conocer la prevalencia de miopía en los estudiantes de medicina de la Universidad de Monterrey, así como estudiar los factores de riesgo asociados. Métodos: Estudio prospectivo, transversal y observacional, realizado entre octubre y diciembre de 2016. Se evaluaron 300 estudiantes de medicina. La presencia de error refractivo se evaluó mediante un autoquerato refractómetro, y la presencia de factores de riesgo conocidos de miopía se analizó mediante un cuestionario. Resultados: 189 alumnos (68.7\%) tuvieron miopía en algún ojo y 149 (54.2\%), en ambos ojos. De los factores estudiados, solo en dos hubo una diferencia estadísticamente significativa: los pacientes con miopía tuvieron mayor edad que los que no la presentaron (21.60 \pm 2.27 vs. $20.77 \pm 2.61$, respectivamente), con una $p=0.01$, además del antecedente de todos los hermanos usuarios de lentes (29.1 vs. $17.4 \%$, respectivamente), con una $p=0.04$. Las horas de lectura, el uso de computadora, el tiempo de ejercicio, el sobrepesolobesidad, el tener padres o algún hermano que utilizan lentes fueron similares en ambos grupos. Conclusiones: En este grupo de alumnos de medicina, la prevalencia de miopía basada en una refracción sin ciploplegia es más alta que en otros grupos étnicos del continente americano, y los únicos factores de riesgo asociados fueron la edad y el que todos los hermanos utilicen lentes.

Palabras clave: Miopía. Prevalencia. Factores de riesgo. Estudiantes de medicina.

\section{Abstract}

Background and Objective: Myopia has become a public health problem, so it is important to know the prevalence of myopia in different populations, like in medical students who are considered a risk group. Our objective was to know the prevalence of myopia in medical students of the University of Monterrey, as well as to study the associated risk factors.

\footnotetext{
Correspondencia:

*Manuel Garza-Leon

Centro Médico Hidalgo, Cons. 706

Hidalgo, 2425, Poniente

Col. Obispado

Fecha de recepción: 08-01-2019

C.P. 64060 , Monterrey N.L, México

E-mail: manuel.garza@udem.edu

Disponible en internet: 01-09-2019 Rev Mex Oftalmol. 2019;93(5):246-253

www.rmo.com.mx 0187-4519/@ 2019 Sociedad Mexicana de Oftalmología. Publicado por Permanyer México. Este es un artículo Open Access bajo la licencia CC BY-NC-ND (http://creativecommons.org/licenses/by-nc-nd/4.0/).
} 
Methods: A prospective, cross-sectional and observational study was conducted between October and December 2016. Three hundred medical students were evaluated regarding the presence of refractive errors through auto-kerato/refractometer measurement and a questionnaire to analyze the presence of known risk factors for myopia. Results: One hundred eighty-nine students (68.7\%) had myopia in one eye, and 149 (54.2\%) in both. From the factors evaluated, only two were statistically significant. One was that patients with myopia were older than those without it (21.60 $\pm 2.27 \mathrm{vs.} 20.77 \pm 2.61$, respectively; $p=0.01)$, and the second one was history of need of visual correction in all siblings $(29.1 \mathrm{vs.} 17.4 \%$ respectively; $p=0.04)$. Reading time, computer use, exercise time, overweight/obesity, having parents or one sibling who needs visual correction were similar between groups. Conclusions: In this group of medical students, the prevalence of myopia based on a refraction without cycloplegia is higher compared to other demographic groups of the Americas, and the only associated risk factors were age and need of visual correction in all siblings.

Key words: Myopia. Prevalence. Risk factors. Medical students.

\section{Introducción}

La miopía es uno de los trastornos oftalmológicos más frecuentes y se define como un error refractivo esférico causado por una potencia de refracción excesiva en relación con la curvatura corneal y el grosor del cristalino y/o aumento del diámetro anteroposterior del globo ocular, los cuales producen una refracción de la luz a un punto focal por delante de la retina.

Estudios en diferentes partes del mundo ${ }^{1-3}$ han demostrado en las últimas décadas un gran incremento en la prevalencia de miopía, especialmente en los países asiáticos ${ }^{2,4}$, razón por la cual se han realizado múltiples estudios tratando de encontrar los factores que producen estos cambios $2,5-7$.

La etiología de la miopía es multifactorial, diversos estudios han evaluado múltiples factores asociados al desarrollo o progresión de miopía en diferentes poblaciones. Una revisión realizada por Foster, et al., en el $2014^{8}$ concluyó que la miopía es el resultado de una compleja interacción entre la predisposición genéti$\mathrm{ca}^{9,10}$ y otros factores ambientales, como la actividad física $^{11}$, las actividades al aire libre ${ }^{12,13}$, las horas de sueño ${ }^{14}$, el peso y la altura ${ }^{15}$, entre otros. Mirshahi, et al. ${ }^{16}$ observaron que personas con grado universitario presentaban una mayor prevalencia de miopía en comparación con personas con niveles de formación más bajos, mientras que Fernández-Montero, et al. ${ }^{17}$, al llevar a cabo un estudio en una cohorte de selección abierta, llegó a la conclusión que la exposición y uso de la computadora se asocia con el desarrollo y la progresión de la miopía. Estos factores se han confirmado en otros estudios epidemiológicos 8,18 .

Estudios en estudiantes de diversas áreas han demostrado una alta prevalencia de miopía ${ }^{4,19,20}$, posiblemente relacionada con que pasan una buena parte del día en actividades de visión cercana ${ }^{19,20}$, así como el frecuente uso de la computadora y las horas de lectura ${ }^{17}$. Entre los grupos con mayor prevalencia se encuentran los estudiantes de medicina ${ }^{4,18,20-26}$. Esto puede ser consecuencia de que pasan una gran cantidad de horas leyendo, realizando trabajos de visión cercana y usando aparatos electrónicos ${ }^{17}$. Debido a ello, los estudiantes de medicina se consideran una población con alta predisposición a la miopía ${ }^{21}$.

Hasta nuestro conocimiento, no existe información en México sobre la prevalencia de errores refractivos en una población estudiantil, además, no se ha confirmado que los factores de riesgo descritos en otras poblaciones son similares a los de nuestra población, por lo que el objetivo de nuestro trabajo fue conocer la prevalencia de miopía en estudiantes de medicina de la Universidad de Monterrey, así como estudiar los factores de riesgo para el desarrollo de la enfermedad.

\section{Material y métodos}

Se realizó un estudio prospectivo, transversal y observacional entre octubre y diciembre de 2016, en el que se evaluó el error refractivo, así como los factores de riesgo asociados mediante un cuestionario, que incluía familiares con ametropías (se definió como el uso de lentes para visión lejana y/o antecedente de cirugía refractiva), lugar que ocupa en su familia, enfermedades oculares, horas de actividad física, horas de lectura a la semana y horas de uso de la computadora a la semana, con un total de 24 preguntas; además, se midió la altura y el peso (Báscula Beurer BG-17), y se calculó el índice de masa corporal (IMC). La medición del error refractivo se realizó de forma automatizada con el uso de un autoquerato refractómetro (Topcon RM-A7000, Topcon Co., Tokio, Japón) sin el uso de gotas ciclopléjicas. Se tomó como el error refractivo al promedio de las 3 tomas del equipo.

Se definió miopía como el equivalente esférico de -0.50 dioptrías(D). La miopía alta se definió como un 
error de refracción igual o mayor a -6.0 D. Se definió sobrepeso/obesidad como un IMC mayor a $24.9 \mathrm{~kg} / \mathrm{m}^{2}$.

Previo al inicio del estudio, se obtuvo la aprobación de las autoridades y el Comité de Ética de la Universidad de Monterrey, y el estudio se adhirió a los principios de la Declaración de Helsinki. Se obtuvo el consentimiento informado de todos los participantes después de explicarles el estudio.

\section{Tamaño de la muestra}

La población objetivo fueron los estudiantes de medicina de la Universidad de Monterrey. Se calculó el tamaño de la muestra mediante una fórmula de comparación de proporciones independientes, de acuerdo a la proporción promedio $(57 \%)$ descrita en tres estudios en diferentes países ${ }^{27-31}$, con una tasa de error del $5 \%$ y un intervalo de confianza del 95\% (IC 95\%), lo que dio un total de 274 personas.

\section{Muestreo}

Se realizó un muestreo estratificado de acuerdo al semestre que cursan los estudiantes, con una selección aleatoria y proporcional dividida por la proporción esperada.

\section{Criterios de inclusión y exclusión}

Se incluyeron a todos los alumnos matriculados en el semestre de otoño de 2016 en la Universidad de Monterrey que aceptaran participar en el estudio y firmaran un consentimiento informado. Se excluyeron a todos los alumnos que tuvieran antecedente de cualquier procedimiento quirúrgico que pudiera modificar el estado refractivo del ojo (cirugía refractiva, cirugía de catarata, cirugía de retina), que padecieran una infección ocular activa o que no cooperaran para el estudio.

\section{Análisis estadístico}

Los datos se analizaron utilizando un programa estadístico (SPSS v21.0; IBM, Chicago, Illinois, EE. UU.). El equivalente esférico del error de refracción se calculó como la suma del defecto esférico más la suma de la mitad del error de refracción cilíndrico calculado en negativo. La prevalencia fue calculada como el número de participantes con el tipo particular de error de refracción en relación con el número total de examinados, y se reportó su media \pm su desviación estándar, así como su IC 95\%. La distribución normal de parámetros fue probado por la prueba de Kolmogorov-Smirnov. En el caso de parámetros no distribuidos normalmente, se utilizó la prueba de Mann-Whitney para examinar la significancia estadística de la diferencia entre los grupos. La prueba $\chi^{2}$ se usó para comparar proporciones. El análisis de regresión lineal se aplicó para examinar asociaciones entre error de refracción y los factores de riesgo estudiados. Se utilizó la regresión logística para comparar la prevalencia de la miopía y todos los factores estudiados juntos. Se calculó el riesgo relativo y su IC $95 \%$. Se consideraron estadísticamente significativos los resultados cuando el valor de $p$ fue menor de 0.05 .

\section{Resultados}

Se evaluaron 275 estudiantes con una edad promedio de $21.34 \pm 2.41$ (rango 18 a 37 años). El $56.4 \%$ de los alumnos (155) fueron del sexo masculino. La tabla 1 muestra los datos demográficos y de factores de riesgo de la población estudiada. Los alumnos con miopía fueron de mayor edad que los alumnos que no la presentaron, de una forma estadísticamente significativa $(p=0.01)$.

Del total de la muestra, el equivalente esférico (EE) del ojo derecho fue $-0.96 \pm 1.27 \mathrm{D}$ (rango $-6.00 \mathrm{a}$ +2.00 ) y del ojo izquierdo, $-0.94 \pm 1.35 \mathrm{D}$ (rango -6.00 $\mathrm{a}+2.50)$. Tuvieron miopía ( $E E \leq-0.50 \mathrm{D}$ ) en alguno de Ios ojos 189 alumnos (68.7\%; IC 95\%: 63.2-74.2\%), y 149 (54.2\%) tuvieron miopía en ambos ojos. El 61.5\% tuvo miopía en el ojo derecho (EE $\leq-0.50 \mathrm{D})$ y el $58.9 \%$, en el ojo izquierdo. La miopía alta ( $E E \leq-6.00$ D) se encontró en seis alumnos (2.18\%), uno de forma bilateral y cinco solo en el ojo izquierdo $(0.4 \%$ de los ojos derechos y $2.2 \%$ de los ojos izquierdos). Los tipos de graduación encontrados se observan en la figura 1.

De los factores de riesgo estudiados (la prevalencia de miopía por género, ser primogénito, tener alguno de los padres o al menos un hermano que utilizaran lentes, padecer enfermedades oculares o presentar obesidad), ninguno tuvo una diferencia estadísticamente significativa, como se demuestra en la tabla 1. Sin embargo, los alumnos en los que todos sus hermanos utilizaban lentes tuvieron una mayor prevalencia de miopía ( $p=0.04$ ), no así cuando se exploró si ambos padres usaban lentes (Tabla 1).

El peso promedio de los participantes fue de $71.82 \pm$ $18.01 \mathrm{~kg}$ (rango 42 a $138 \mathrm{~kg}$ ); la altura promedio, de $169.49 \pm 9.13 \mathrm{~cm}$ (rango $149 \mathrm{a} 195 \mathrm{~cm}$ ), y el IMC promedio, de $24.77 \mathrm{mg} / \mathrm{kg}^{2} \pm 4.69$ (rango 16.24 a $46.65 \mathrm{mg} / \mathrm{kg}^{2}$ ). 
Tabla 1. Datos demográficos y factores de riesgo de la población estudiada

\begin{tabular}{|c|c|c|c|c|}
\hline Variable & Total $(n=275)$ & Alumnos con miopía ( $n=189$ ) & Alumnos sin miopía ( $n=86$ ) & Valor p \\
\hline Edad años & $21.34 \pm 2.41$ & $21.60 \pm 2.27$ & $20.77 \pm 2.61$ & 0.01 \\
\hline Sexo m/f, n (\%) & $155 / 120(56.4 / 43.6)$ & $106 / 83(56.1 / 43.9)$ & 49/37 (57/43) & 0.89 \\
\hline Primogénito, n (\%) & $136(49.5)$ & $94(49.7)$ & $42(48.8)$ & 0.89 \\
\hline $\begin{array}{l}\text { Algún hermano con } \\
\text { lentes, } \mathrm{n}(\%)\end{array}$ & $148(53.8)$ & $106(56.1)$ & $42(48.8)$ & 0.26 \\
\hline $\begin{array}{l}\text { Todos los hermanos con } \\
\text { lentes, } \mathrm{n}(\%)\end{array}$ & $70(25.5)$ & $55(29.1)$ & $15(17.4)$ & 0.04 \\
\hline $\begin{array}{l}\text { Alguno de los padres } \\
\text { con lentes, } n(\%)\end{array}$ & $204(74.2)$ & $140(74.1)$ & $64(74.4)$ & 0.95 \\
\hline $\begin{array}{l}\text { Ambos padres con } \\
\text { lentes, } \mathrm{n}(\%)\end{array}$ & $137(49.8)$ & $95(50.3)$ & $42(48.8)$ & 0.82 \\
\hline $\begin{array}{l}\text { Enfermedades oculares, } \\
\text { n (\%) }\end{array}$ & $11(4)$ & $7(3.7)$ & $4(4.7)$ & 0.71 \\
\hline Horas de lectura* & $18.22 \pm 15.95$ & $18.63 \pm 16.90$ & $17.34 \pm 13.71$ & 0.53 \\
\hline $\begin{array}{l}\text { Horas de uso de } \\
\text { computadora* }\end{array}$ & $36.29 \pm 26.83$ & $37.14 \pm 28.821$ & $34.42 \pm 23.59$ & 0.43 \\
\hline Obesidad, \% & 40.4 & 42.9 & 34.9 & 0.21 \\
\hline Horas de ejercicio* & $3.77 \pm 3.62$ & $3.64 \pm 3.41$ & $4.05 \pm 4.06$ & 0.28 \\
\hline
\end{tabular}

*A la semana

f: femenino; m: masculino.

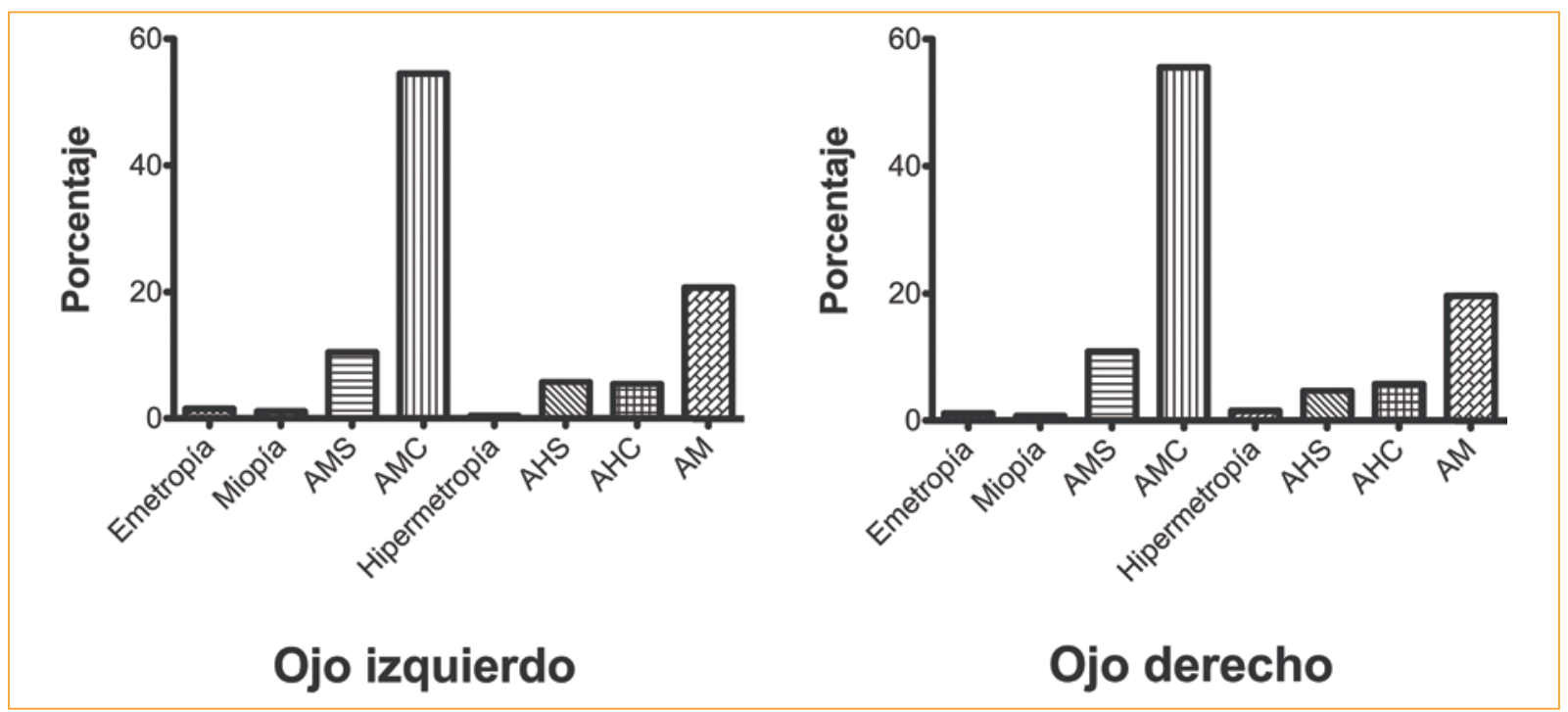

Figura 1. Distribución de errores refractivos en alumnos de medicina.

AHC: astigmatismo hipermetrópico compuesto; AHS: astigmatismo hipermetrópico simple; AM: astigmatismo mixto; AMC: astigmatismo miópico compuesto; AMS: astigmatismo miópico simple.

La tasa de sobrepeso/obesidad en los alumnos fue del $40.4 \%$ (111 alumnos), de los cuales, 81 (73\%) presentaban miopía. De los alumnos sin obesidad, el $65.9 \%$ (108 alumnos) presentaban miopía ( $p=0.212$ ).
Se estudiaron el sexo, ser primogénito, el uso de lentes por parte de alguno de los hermanos, todos los hermanos, alguno de los padres o ambos padres, enfermedades oculares, horas de lectura, uso de computadora, 
obesidad y horas de ejercicio como posibles factores de riesgo en un modelo de regresión logística multivariada ajustada a la edad (Tabla 2).

\section{Discusión}

En este estudio encontramos que la prevalencia de miopía en estudiantes de medicina en Monterrey fue del $68.7 \%$ (IC 95\%: 63.2-74.2\%). Este resultado es mayor al reportado en estudios realizados en países europeos, como el de Midelfart, et al. ${ }^{23}$, quienes en 1989 encontraron una prevalencia del $50.3 \%$ en 133 estudiantes de medicina en Noruega, resultado similar en alumnos en Dinamarca de 1996 a 1998, quienes reportaron un $50 \%$. Un estudio en estudiantes de medicina en Turquía entre 2003 y 2004 encontró una prevalencia aún menor $(32.9 \%)^{18}$. Estudios en otras regiones del mundo han reportado prevalencias generalmente mayores, sobre todo en el continente asiático, donde la prevalencia reportada varía entre el $35.86 \%$ en la India en el año $2014^{27}$, el $53.7 \%$ en Arabia Saudita ${ }^{28}$, el $57.6 \%$ en Paquistán ${ }^{7}$, el $84.1 \%$ en China entre los años 2007 y $2012^{20,29}$, y la mayor prevalencia en Singapur en el año 2000, con el 89.8\% (IC 95\%: 85,94\%) y en Taiwán, con el $95.8 \%$ entre 1987 y $1999^{4}$. Hasta el momento, la información en el continente americano y el Caribe es muy escasa, en Dominica, un estudio de Halari, et al, encontró una prevalencia del $31.9 \%$ en 116 alumnos de medicina ${ }^{30}$, y en Ecuador, del $26.67 \%$ en 180 alumnos en el año $2013^{31}$. Sin embargo, la metodología, las tasas de no participación, las técnicas de refracción difieren, lo que limita para hacer comparaciones.

La edad de los alumnos con miopía fue mayor de forma estadísticamente significativa que la de los alumnos sin miopía (-0.83 años; IC95\%: -0.22 a -1.44). Esto concuerda con otros estudios que han demostrado que, a mayor edad, mayor prevalencia de miopía debido tanto a que la miopía se desarrolla de forma tardía, así como a la progresión de la misma ${ }^{7,23,32}$, lo cual se ha descrito en especial en estudiantes de medicina, como lo reportó Midelfart, et al. en un grupo de alumnos de medicina en Noruega, donde el $43.3 \%$ de los alumnos iniciaron el uso de lentes correctivos a los 19 años o más y, generalmente, no desarrollan miopía alta, lo cual concuerda con nuestros alumnos ${ }^{23}$.

El efecto de las hormonas sexuales en el desarrollo y prevalencia de la miopía ha sido ampliamente estudiado ${ }^{33,34}$. Estudios epidemiológicos han corroborado la mayor prevalencia de miopía en mujeres, sobre todo de miopía alta $^{35}$, mientras que otros estudios han
Tabla 2. Factores de riesgo asociados a la presencia de miopía a través de una regresión logística multivariada

\begin{tabular}{|c|c|c|c|}
\hline Variable & $\begin{array}{l}\text { Riesgo } \\
\text { relativo }\end{array}$ & IC del $95 \%$ & valor $p$ \\
\hline Género & 1.03 & $0.62-1.73$ & 0.89 \\
\hline Primogénito & 1.05 & $0.60-1.81$ & 0.85 \\
\hline $\begin{array}{l}\text { Algún hermano con } \\
\text { lentes }\end{array}$ & 1.04 & $0.55-1.97$ & 0.88 \\
\hline $\begin{array}{l}\text { Todos los hermanos } \\
\text { con lentes }\end{array}$ & 1.91 & $0.89-4.08$ & 0.09 \\
\hline $\begin{array}{l}\text { Alguno de los padres } \\
\text { con lentes }\end{array}$ & 0.85 & $0.41-1.78$ & 0.67 \\
\hline $\begin{array}{l}\text { Ambos padres con } \\
\text { lentes }\end{array}$ & 1.17 & $0.62-2.22$ & 0.62 \\
\hline Enfermedades oculares & 1.01 & $0.60-1.70$ & 0.95 \\
\hline Horas de lectura* & 1.02 & $0.56-1.77$ & 0.99 \\
\hline $\begin{array}{l}\text { Horas de uso de } \\
\text { computadora* }\end{array}$ & 1.09 & $0.62-1.92$ & 0.73 \\
\hline Obesidad & 1.38 & $0.80-2.36$ & 0.24 \\
\hline Horas de ejercicio* & 1.49 & $0.87-2.54$ & 0.13 \\
\hline
\end{tabular}

*Alumnos con más horas de la media

sugerido también un efecto medioambiental, ya que la mayor prevalencia se observa en mujeres blancas y del continente del este asiático a partir de los 9 años de edad ${ }^{36}$. Esta diferencia ha tenido resultados contradictorios en alumnos de medicina, ya que algunos estudios, sobre todo de países asiáticos, reportan tasas diferentes ${ }^{7,20,27,29,30}$, mientras que otros, al igual que el nuestro ( $69.2 \%$ en mujeres vs. $68.4 \%$ en hombres), no encuentran diferencias importantes ${ }^{18,22,23}$. Una de las posibles razones de esto es que en nuestro estudio la prevalencia de miopía alta fue muy baja, ya que solo el $2.18 \%$ de los alumnos la presentaron, y es en este grupo de pacientes donde la prevalencia es mucho mayor en mujeres que en hombres ${ }^{35}$.

El orden de nacimiento ha sido reportado en algunos estudios como un factor de riesgo para desarrollar miopía, ${ }^{5,37-39}$, sin embargo, la asociación reportada es baja $(\mathrm{OR}<1.3)^{5}$, muy similar a la de nuestro estudio (1.33; IC 95\%: 0.80-2.23), aunque en nuestro estudio esta diferencia no fue estadísticamente significativa. Esto podría deberse a lo reportado por Guggenheim, et al., quienes analizaron datos de cuatro estudios epidemiológicos diferentes y encontraron que solo estudios con más de 4,000 participantes proveen un poder estadístico adecuado para apoyar esta asociación ${ }^{5}$, 
número mucho mayor al de nuestro estudio, además Morgan, et al. sugieren que esta asociación podría deberse a un factor de confusión por el grado de educación ${ }^{40}$, ya que algunos estudios reportan una mayor educación en los hijos primogénitos que en sus hermanos $^{6}$, factor que obviamente no está presente en nuestro estudio, ya que todos los sujetos evaluados son estudiantes de universidad.

La influencia de la genética en el desarrollo de la miopía ha sido ampliamente estudiada ${ }^{8-10,18,41-44}$. Se han estudiado las mutaciones de los genes LRPAP1, CTSH, LEPREL143,44, ZNF644, SLC39A5, y SCO2 en China ${ }^{44}$ y su relación con la miopía alta. En nuestro estudio, la influencia genética medida a través de la prevalencia de padres y hermanos con miopía no demostró una relación estadísticamente significativa. En el caso de los padres, se compara con un estudio realizado en China en estudiantes universitarios y sus padres, que arroja un resultado estadísticamente significativo cuando los padres presentan miopía ${ }^{45}$, sin embargo, en el caso de nuestro estudio, no presentan la misma relación. Aunque este mismo estudio no descarta la influencia de factores ambientales que podrían influir en el desarrollo de la enfermedad.

En nuestro estudio encontramos que la prevalencia de miopía era mayor cuando todos los hermanos usaban lentes, este resultado es similar al encontrado por Czepita, et al., realizado en Polonia ${ }^{42}$, quienes estudiaron 5,533 estudiantes con material y métodos muy similares a los de nuestro estudio, y obtuvieron resultados estadísticamente significativos para la relación entre la frecuencia de miopía en los estudiantes y el hecho de que sus padres o hermanos padezcan la enfermedad $(p<0.001)$.

Se ha estudiado en diversos países la relación que existe entre el ejercicio y el desarrollo de miopía, con resultados contradictorios ${ }^{11,12,46,47}$. En Australia ${ }^{46}$ un estudio a un grupo de estudiantes universitarios de entre 18 y 25 años no encontró una correlación importante entre el ejercicio y la cantidad del mismo con la presencia y gravedad de la miopía. Fueron los mismos resultados obtenidos en el caso de nuestro estudio, sin embargo, la muestra del estudio australiano era pequeña (27 sujetos), y esto es un factor que puede alterar los resultados. Por otra parte, un estudio longitudinal de cohorte de 2 años de duración que evaluó la actividad física como factor protector en el desarrollo de la enfermedad en Dinamarca ${ }^{11}$, con enfoque en estudiantes de medicina, concluyó que existe esta relación como factor protector. Otros estudios ${ }^{47}$ han tenido resultados similares al nuestro.
En nuestro estudio se encontró que el uso de la computadora por encima de la media $(36.29 \pm 26.82 \mathrm{~h}$ a la semana) no tuvo una relación directa con la presencia de miopía. Esto contrasta con el estudio de cohorte realizado por Fernández-Montero, et al. en $2015^{17}$, en donde se evaluaron 17,217 estudiantes universitarios de España, con un uso medio de la computadora de $14.3 \mathrm{~h} /$ semana, y encontraron una relación entre la exposición a la computadora y el desarrollo y/o progresión de la miopía. Esto a pesar de que en nuestro estudio los estudiantes pasaron una mayor cantidad de horas utilizando la computadora que en el estudio mencionado, sin embargo, el tamaño de la muestra es muy superior al nuestro.

Algunos reportes en la literatura han documentado la relación entre el grado de escolaridad y las actividades de visión cercana, principalmente la lectura, por lo que hay múltiple evidencia que respalda al grado de escolaridad $^{3,16}$, nivel socioeconómico alto ${ }^{48}$ y mayor cantidad de actividades de visión cercana ${ }^{49}$ como factores de riesgo para la miopía, no obstante, los mecanismos exactos sobre el cómo se favorece el desarrollo de esta enfermedad permanecen controversiales. En un estudio realizado en población australiana infantil, Ip, et al. encontraron que el mayor tiempo de lectura realizada a corta distancia $(<30 \mathrm{~cm})$ se asoció con mayor prevalencia de miopía, incluso después de ajustarse por edad, sexo, etnia y escolaridad $(p=0.02$ y $p$ $=0.0003$, respectivamente $)^{50}$. En cuanto a las horas de lectura, en nuestros participantes, el 33.1\% (91) se encontraban por encima de la media, que fue de $18.22 \pm$ $15.95 \mathrm{~h}$ a la semana, y de forma similar, un $38.9 \%$ (107) pasaban más de $40 \mathrm{~h}$ frente a un aparato electrónico, considerando la lectura también de esta forma.

Se ha estudiado si las características antropométricas (peso, talla e IMC) favorecen el desarrollo de la miopía ${ }^{15,51-53}$,. En nuestro estudio no hubo una relación entre estas y la presencia de miopía, dato similar a lo reportado por otros autores ${ }^{51,52}$. Uno de de los estudios con un mayor tamaño de la muestra, realizado por Rosner, et al..$^{51}$ en más de 100,000 hombres entre 17 y 19 años, encontró que la miopía no se asociaba con la talla alta ni mayor peso, dado que las personas con miopía alta tenían un IMC menor comparadas con los que sufrían de miopía moderada o no la presentaban. Igualmente. Saw SM, et al. ${ }^{52}$ estudiaron la asociación entre las medidas antropométricas de niños en China y los parámetros oculares y su refracción, y encontraron que los niños con mayor IMC tendían más a la refracción hipermetrópica. En nuestro estudio no hubo una relación entre sobrepeso/obesidad y miopía. 
Nuestro estudio tiene algunas limitaciones, la más importante es la medición del defecto refractivo por autorrefractometría sin usar gotas ciclopléjicas, sin embargo, esta es una práctica usual en estudios poblacionales en adultos ${ }^{35}$, y existe controversia sobre el efecto de la acomodación en la medición no ciclopléjica de la refracción en adultos, ya que algunos estudios reportan que aun en adultos existe un error en la medición de la graduación ${ }^{54-56}$, generalmente es menor a $0.50 D^{56}$; mientras otros estudios, como el de Sanfilippo, et al. ${ }^{57}$, sugieren que en adultos de 20 años o más no es necesario realizarla, y nuestro mayor grupo de alumnos se encuentra en ese rango de edad. Por otra parte, para evaluar el efecto del uso de lentes en padres y hermanos se utilizó solo un cuestionario, por lo que podría perderse un porcentaje de pacientes, que aun y cuando tienen un error refractivo no utilizan lentes, y puede ser importante como lo reportó Bai, et al. en un estudio en China en 2014 en que se estimó que de los pacientes que necesitan lentes, el $34 \%$ no los usan ${ }^{58}$. Finalmente, el estudio solo evaluó alumnos universitarios que estudian medicina, y aunque otros estudios han demostrado tasas similares entre los alumnos de medicina ${ }^{35,45} y$ de otras carreras ${ }^{19,59}$, no es posible generalizar nuestros resultados a todo estudiante universitario.

\section{Conclusiones}

En conclusión, en este grupo de alumnos de medicina de la Universidad de Monterrey, la prevalencia de miopía basada en una refracción sin ciploplegia es más alta que en otros grupos étnicos del continente americano, y los únicos factores de riesgo asociados fueron la edad y el que todos los hermanos utilicen lentes.

\section{Agradecimiento}

Nuestro agradecimiento a los estudiantes Montserrat de la Garza, César Gustavo Mendoza Lara, José Iván López Haces y José Julio Villarreal Tule por su ayuda para la medición del error refractivo de los alumnos.

\section{Conflicto de intereses}

Los autores declaran que no existe conflicto de intereses.

\section{Responsabilidades éticas}

Protección de personas y animales. Los autores declaran que los procedimientos seguidos se conformaron a las normas éticas del comité de experimentación humana responsable y de acuerdo con la Asociación Médica Mundial y la Declaración de Helsinki.

Confidencialidad de los datos. Los autores declaran que han seguido los protocolos de su centro de trabajo sobre la publicación de datos de pacientes.

\section{Derecho a la privacidad y consentimiento infor-} mado. Los autores han obtenido el consentimiento informado de los pacientes y/o sujetos referidos en el artículo. Este documento obra en poder del autor de correspondencia.

\section{Bibliografía}

1. Guo L, Yang J, Mai J, Du X, Guo Y, Li P, et al. Prevalence and associated factors of myopia among primary and middle school-aged students: a school-based study in Guangzhou. Eye (Lond.). 2016;30(6):796-804.

2. Lee YY, Lo CT, Sheu SJ, Yin LT. Risk factors for and progression of myopia in young Taiwanese men. Ophthalmic Epidemiol. 2015;22(1):66-73.

3. Williams KM, Bertelsen G, Cumberland P, Wolfram C, Verhoeven VJ, Anastasopoulos E, et al. Increasing Prevalence of Myopia in Europe and the Impact of Education. Ophthalmology. 2015;122(7):1489-97.

4. Lin LL, Shih YF, Lee YC, Hung PT, Hou PK. Changes in ocular refraction and its components among medical students--a 5-year longitudinal study. Optom Vis Sci. 1996;73(7):495-8.

5. Guggenheim JA, McMahon G, Northstone K, Mandel Y, Kaiserman I, Stone RA, et al. Birth order and myopia. Ophthalmic Epidemiol. 2013; 20(6):375-84

6. Booth AL, Kee HJ. Birth Order Matters: The Effect of Family Size and Birth Order on Educational Attainment. J Popul Econ. 2009;22(2):367-97.

7. Chaudhry R, Ali $\mathrm{H}$, Sheikh NH. Frequency and underlying factors of myopia among medical students. Biomedica. 2011;27(2):154-60.

8. Foster PJ, Jiang Y. Epidemiology of myopia. Eye (Lond). 2014;28(2):202-8.

9. Kurtz D, Hyman L, Gwiazda JE, Manny R, Dong LM, Wang Y, et al. Role of parental myopia in the progression of myopia and its interaction with treatment in COMET children. Invest Ophthalmol Vis Sci. 2007; 48(2):562-70

10. Lim LT, Gong Y, Ah-Kee EY, Xiao G, Zhang X, Yu S. Impact of parental history of myopia on the development of myopia in mainland china school-aged children. Ophthalmol Eye Dis. 2014;6:31-5.

11. Jacobsen $N$, Jensen $H$, Goldschmidt $E$. Does the level of physical activity in university students influence development and progression of myopia?--a 2-year prospective cohort study. Invest Ophthalmol Vis Sci. 2008;49(4):1322-7.

12. Jin JX, Hua WJ, Jiang $X$, Wu XY, Yang JW, Gao GP, et al. Effect of outdoor activity on myopia onset and progression in school-aged children in northeast China: the Sujiatun Eye Care Study. BMC Ophthalmol. 2015;15:73.

13. McKnight CM, Sherwin JC, Yazar S, Forward H, Tan AX, Hewitt AW et al. Myopia in young adults is inversely related to an objective marker of ocular sun exposure: the Western Australian Raine cohort study. Am J Ophthal. 2014;158(5):1079-85.

14. Jee D, Morgan IG, Kim EC. Inverse relationship between sleep duration and myopia. Acta Ophthalmol. 2015;94(3):e204-10.

15. Rim TH, Kim SH, Lim KH, Kim HY, Baek SH. Body Stature as an Age-Dependent Risk Factor for Myopia in a South Korean Population. Semin Ophthalmol. 2017;32(3):326-36

16. Mirshahi A, Ponto KA, Hoehn R, Zwiener I, Zeller T, Lackner K, et al. Myopia and level of education: results from the Gutenberg Health Study. Ophthalmology. 2014;121(10):2047-52.

17. Fernandez-Montero A, Olmo-Jimenez JM, Olmo N, Bes-Rastrollo M, Moreno-Galarraga $\mathrm{L}$, Moreno-Montanes $\mathrm{J}$, et al. The impact of computer use in myopia progression: a cohort study in Spain. Prev Med. 2015;71:67-71.

18. Onal S, Toker E, Akingol Z, Arslan G, Ertan S, Turan C, et al. Refractive errors of medical students in Turkey: one year follow-up of refraction and biometry. Optom Vis Sci. 2007;84(3):175-80.

19. Loman J, Quinn GE, Kamoun L, Ying GS, Maguire MG, Hudesman D, et al. Darkness and near work: myopia and its progression in third-year law students. Ophthalmology. 2002;109(5):1032-8.

20. Lv L, Zhang Z. Pattern of myopia progression in Chinese medical students: a two-year follow-up study. Graefe's archive for clinical and experimental ophthalmology = Albrecht von Graefes Archiv fur klinische und experimentelle Ophthalmologie. 2013;251(1):163-8.

21. Riegelman RK. Medical Student Myopia Syndrome: a recently recognized pan-epidemic. Am J Prev Med. 1991;7(4):252. 
22. Woo W, Lim K, Yang $H$, Lim X, Liew F, Lee $Y$, et al. Refractive errors in medical students in Singapore. Singapore Med J. 2004;45:470-4.

23. Midelfart A, Aamo B, Sjohaug KA, Dysthe BE. Myopia among medical students in Norway. Acta Ophthalmol (Copenh). 1992;70(3):317-22.

24. Mozolewska-Piotrowska K, Stepniewska J, Nawrocka J. [Frequency and incidence of myopia among medical students]. Klinika Oczna. 2005 107(7-9):468-70.

25. Akhanda AH, Quayum MA, Siddiqui NI, Hossain MM. Refractive status of medical students of mymensingh medical college. Mymensingh Med J. 2010;19(4):493-6.

26. Al-Rashidi $\mathrm{SH}$, Albahouth $\mathrm{AA}$, Althwini WA, Alsohibani $\mathrm{AA}$ Alnughaymishi AA, Alsaeed AA, et al. Prevalence Refractive Errors among Medical Students of Qassim University, Saudi Arabia: Cross-Sectional Descriptive Study. Open Access Maced J Med Sci. 2018:6(5):940-3.

27. Dey AK, Chaudhuri SK, Jana S, Ganguly P, Ghorai S, Sarkar A. Prevalence of refractive errors in medical students. Int J Health Sci Res. 2014;4(8):98-102.

28. Al-Rashidi SH, Albahouth AA, Althwini WA, Alsohibani AA, Alnughaymishi AA, Alsaeed AA, et al. Prevalence Refractive Errors among Medical Students of Qassim University, Saudi Arabia: Cross-Sectional Descriptive Study. Open Access Macedonian J Med Sci. 2018;6(5):940.

29. Wang L, Du M, Yi H, Duan S, Guo W, Qin P, et al. Prevalence of and Factors Associated with Myopia in Inner Mongolia Medical Students in China, a cross-sectional study. BMC Ophthalmology. 2017;17(1):52.

30. Halari MM, Halari CD, Adeiza OD, Calista IU, Ayotunde AH. Prevalence of Eye Defects Among Medical Students in Dominica. ASRJETS. 2016; 18(1):133-41.

31. Arellano G, Chávez A, Arellano S, Chaves C. Estudio de la agudeza visual y problemas refractivos en estudiantes de medicina de la escuela superior politécnica de chimborazo 2013. SCientifica. 2014;12:25.

32. Kinge B, Midelfart A, Jacobsen G, Rystad J. The influence of near-work on development of myopia among university students. A three-year longitudinal study among engineering students in Norway. Acta Ophthalmologica Scandinavica. 2000;78(1):26-9.

33. Zhu X, Lin T, Stone RA, Laties AM. Sex differences in chick eye growth and experimental myopia. Exp Eye Res. 1995;61(2):173-9.

34. Xie H, Mao X, Yang H, Xie Z, Pan Y, Gao Y. [Analysis on the relationship between adolescent myopia and serum sex hormone]. Zhonghua $Y$ Xue Za Zhi. 2014;94(17):1294-7.

35. Hirsch MJ. Sex differences in the incidence of various grades of myopia. Am J Optom Arch Am Acad Optom. 1953;30(3):135-8.

36. Rudnicka AR, Kapetanakis VV, Wathern AK, Logan NS, Gilmartin B, Whincup $\mathrm{PH}$, et al. Global variations and time trends in the prevalence of childhood myopia, a systematic review and quantitative meta-analysis: implications for aetiology and early prevention. $\mathrm{Br} J$ Ophthalmol. 2016;100(7):882-90.

37. Bar Dayan Y, Levin A, Morad Y, Grotto I, Ben-David R, Goldberg A, et al. The changing prevalence of myopia in young adults: a 13-year series of population-based prevalence surveys. Invest Ophthalmol Vis Sci. 2005;46(8):2760-5

38. Mandel Y, Grotto I, El-Yaniv R, Belkin M, Israeli E, Polat U, et al Season of birth, natural light, and myopia. Ophthalmology. 2008;115(4):686-92.

39. Zylbermann R, Landau D, Berson D. The influence of study habits on myopia in Jewish teenagers. J Pediatr Ophthalmol Strabismus. 1993;30(5):319-22.

40. Morgan IG, Cotch MF. Birth order and myopia: what are the messages to readers? Ophthalmic Epidemiol. 2013;20(6):333-4

41. Wenbo L, Congxia B, Hui L. Genetic and environmental-genetic interaction rules for the myopia based on a family exposed to risk from a myopic environment. Gene. 2017;626:305-8.
42. Czepita D, Mojsa A, Ustianowska M, Czepita M, Lachowicz E. The effect of genetic factors on the occurrence of myopia. Klinika Oczna. 2011; 113(1-3):22-4.

43. Feng $C Y$, Huang $X Q$, Cheng $X W$, Wu RH, Lu F, Jin ZB. Mutational screening of SLC39A5, LEPREL1 and LRPAP1 in a cohort of 187 high myopia patients. Scientific Reports. 2017;7(1):1120.

44. Jiang $D, L i J$ J Xiao X, Li S, Jia X, Sun W, et al. Detection of mutations in LRPAP1, CTSH, LEPREL1, ZNF644, SLC39A5, and SCO2 in 298 families with early-onset high myopia by exome sequencing. Invest Ophthalmol Vis Sci. 2014;56(1):339-45.

45. Guo K, Yang da Y, Wang Y, Yang XR, Jing XX, Guo YY, et al. Prevalence of myopia in schoolchildren in Ejina: the Gobi Desert Children Eye Study. Invest Ophthalmol Vis Sci. 2015;56(3):1769-74.

46. Battersby K, Koy L, Phillips N, Sim J, Wilk J, Schmid KL. Analysis of physical activity in emmetropic and myopic university students during semester and holiday periods: a pilot study. Clin Exper Optom. 2015; 98(6):547-54.

47. Muhamedagic L, Alajbegovic-Halimic J, Muhamedagic B, Muracevic B. Relation between physical activity and myopia progression in student population.Med Glas (Zenica). 2013;10(2):385-90.

48. Rudnicka AR, Owen CG, Richards M, Wadsworth ME, Strachan DP. Effect of breastfeeding and sociodemographic factors on visual outcome in childhood and adolescence. Am J of Clin Nutr. 2008;87(5):1392-9.

49. Ku PW, Steptoe A, Lai YJ, Hu HY, Chu D, Yen YF, et al. The Associations between Near Visual Activity and Incident Myopia in Children: A Nationwide 4-Year Follow-up Study. Ophthalmology. 2019;126(2):214-20.

50. Ip JM, Saw SM, Rose KA, Morgan IG, Kifley A, Wang JJ, et al. Role of near work in myopia: findings in a sample of Australian school children. Invest Ophthal Vis Sci. 2008:49(7):2903-10.

51. Rosner M, Laor A, Belkin M. Myopia and stature: findings in a population of 106,926 males. Eur J Ophthalmol. 1995;5(1):1-6.

52. Saw SM, Chua WH, Hong CY, Wu HM, Chia KS, Stone RA, et al. Height and its relationship to refraction and biometry parameters in Singapore Chinese children. Invest Ophthalmol Vis Sci. 2002;43(5):1408-13.

53. Erna R, Husin S, Harahap DH, Randana MPC. Correlation between Body Mass Index and Myopia in Medical Student Universitas Sriwijaya Palembang Indonesia. J Res Med Dent Sci. 2017;5(6):30-2.

54. Hashemi H, Khabazkhoob M, Asharlous A, Soroush S, Yekta A, Dadbin N, et al. Cycloplegic autorefraction versus subjective refraction: the Tehran Eye Study. Br J Ophthalmol. 2016;100(8):1122-7.

55. Fotouhi A, Morgan IG, Iribarren R, Khabazkhoob M, Hashemi H. Validity of noncycloplegic refraction in the assessment of refractive errors: the Tehran Eye Study. Acta Ophthalmologica. 2012;90(4):380-6.

56. Mimouni M, Zoller L, Horowitz J, Wygnanski-Jaffe T, Morad Y, Mezer E. Cycloplegic autorefraction in young adults: is it mandatory? Graefe's archive for clinical and experimental ophthalmology. Albrecht von Graefes Archiv fur Klinische und Experimentelle Ophthalmologie. 2016; 254(2):395-8.

57. Sanfilippo PG, Chu BS, Bigault O, Kearns LS, Boon MY, Young TL, et al. What is the appropriate age cut-off for cycloplegia in refraction? Acta Ophthalmologica. 2014;92(6):e458-62.

58. Bai Y, Yi H, Zhang L, Shi Y, Ma X, Congdon N, et al. An investigation of vision problems and the vision care system in rural china. Southeast Asian J Trop Med Public Health. 2014;45(6):1464-73.

59. Zadnik K, Mutti DO. Refractive error changes in law students. Am J Optom Physiol Opt. 1987;64(7):558-61. 